\title{
SUPPLEMENTARY
}

\section{Scattering models}

\section{Microemulsion Droplets}

The scattering intensity $I_{m E}(q)$ of all experiments in the main paper was described using a form factor of polydisperse spherical or elongated core-shell droplets $\left(P_{\text {sphere }}(q) \text { or } P_{\text {cylinder }}(q)\right)^{1-3}$. The extracted structural parameters are the mean radius $R_{0}$ (for elongated droplets: cross section $R_{0}$ and length $L$ ), polydispersity $\sigma_{0} / R_{0}$, shell thickness $d$ and shape of the radial density distribution function $\chi$. To account for interparticle interactions the polydisperse Percus-Yevick structure factor $\left(S_{\mathrm{PY}}(q)\right)$ was used with the hard sphere interaction radius $R_{\mathrm{HS}}$, its polydispersity $\sigma_{\mathrm{HS}} / R_{\mathrm{HS}}$, and the volume fraction $\phi_{\mathrm{HS}}$ being the fit parameters ${ }^{4}$. Applying the decoupling approximation the scattering intensity $I_{m E}(q)$ is then given by

$$
I_{m E}(q)=n \cdot P(q) \cdot S(q)
$$

with $n$ being the particle number density. In the following the separate scattering contributions used will be discussed briefly, for more information the reader is referred to the original literature.

Form factors:

Spherical droplets:

In case of spherical droplets the particle number density can be defined as 


$$
n=\phi_{C, i} \frac{a_{C}}{4 \pi v_{C}\left(R_{0}^{2}+\sigma_{0}^{2}\right)}
$$

with $\phi_{C, i}$ being the volume fraction of surfactant in the interface, $v_{\mathrm{C}}$ and $a_{\mathrm{C}}$ being the volume and head group area per surfactant molecule, $R_{0}$ being the average radius and $\sigma_{0}$ its polydispersity.

The particle form factor is given by the product of the squared particle volume $V_{\text {part }}$ and the complex square of the normalized scattering amplitude

$$
A(q)=\frac{4 \pi}{V_{\text {part }}} \int_{0}^{\infty} d r \Delta \rho(r) r^{2} \frac{\sin (q r)}{q r}
$$

for spherical particles with a radial scattering length density profile $\Delta \rho(r)$ that can be related to the radial density distribution function $f(r, R)$ by $\Delta \rho(r)=f(r, R) \cdot \Delta \rho(r)_{\text {film. }}$. The radial scattering length density distribution function allows to differentiate between scattering contributions of the surfactant film and the droplet core and is given by

$$
f_{\text {droplet }}(r, R)=\frac{1}{\exp \left(\frac{r-R-d / 2}{\chi}\right)+1}-\frac{1-\Delta \rho_{\text {core }} / \Delta \rho_{\text {film }}}{\exp \left(\frac{r-R+d / 2}{\chi}\right)+1}
$$

according to Foster ${ }^{1} . \Delta \rho_{\text {core }}$ and $\Delta \rho_{\text {film }}$ denote the scattering length density difference between core and solvent and film and solvent, respectively. The thickness of the amphiphilic film is given by the parameter $d$ and the diffusity of the profile is described by $\chi$. To account for polydispersity of the particle radius the form factor is convoluted with a Gaussian size distribution

$$
W\left(R, R_{0}, \sigma_{0}\right)=\frac{1}{\sqrt{2 \pi \sigma_{0}^{2}}} \exp \left(-\frac{\left(R-R_{0}\right)^{2}}{2 \sigma_{0}^{2}}\right)
$$


centered around the average radius $R_{0}$. Combining these terms yields the scattering form factor as:

$$
\bar{P}_{\text {droplet }}(q)=\int_{0}^{\infty} P(q, R) \cdot W\left(R, R_{0}, \sigma_{0}\right) d R
$$

with $P(q, R)$ being the complex square of $A(q)$.

\section{Elongated droplets}

Following ${ }^{2,5}$ in the limit of $q R \ll 1$ the form factor of an elongated droplet or cylinder $P_{\text {cylinder }}(q)$ can approximated by factorization into an axial $\left(P_{\operatorname{rod}}(q)\right)$ and a radial or cross sectional contribution $\left(P_{\text {cross }}(q)\right)$ via

$$
\begin{gathered}
P_{\text {cylinder }}(q)=L_{0}^{2} \int_{0}^{\infty} d L P_{\text {rod }}(q) W_{L}\left(L, L_{0}, \sigma_{L}\right) \\
\int_{0}^{\infty} d R P_{\text {cross }}(q) W_{R}\left(R, R_{0}, \sigma_{R}\right)
\end{gathered}
$$

with $L_{0}$ and $R_{0}$ being the average length and cross section radius of the cylinder, respectively.

The axial contribution is given by

$$
P_{\text {rod }}(q)=\frac{2 \operatorname{Si}(q L)}{q L}-\frac{4 \sin ^{2}(0.5 q L)}{(q L)^{2}}
$$

with $\operatorname{Si}(q L)$ being the sine integral of $q L$. Describing the cross sectional term the core and film scattering contribution can be accounted for. The cross sectionial form factor is then given by

$$
P_{\text {cross }}(q)=\left[2 \pi \Delta \rho_{\text {film }} \int_{0}^{\infty} r f_{\text {droplet }}(r, R) J_{0}(q r) d r\right]^{2}
$$


with $J_{0}(q r)$ being the zeroth order Bessel function of the first kind.

In contrast to the spherical description above the particle number density is defined by

$$
n=\phi_{c, i} \frac{a_{C}}{2 \pi v_{C} R_{0}\left(R_{0}+L_{0}\right)} .
$$

This model results in the fit parameters being the cylinders length $L_{0}$, the cross section radius $R_{0}$ and their respective polydispersities $\sigma_{\mathrm{i}}$. The surfactant film is furthermore described by its thickness $d$ and its diffusity $\chi$.

Percus-Yevick structure factor

To account for repulsive interactions between single particles the structure factor calculated according to Percus-Yevick was used. ${ }^{4}$ It describes a repulsive hard sphere potential and is related to the Fourier transform of the direct correlation function $c(q)$ via

$$
S_{P Y}(q)=\frac{1}{1-n c(q)}
$$

with $n$ being the particle number density. The correlation function is given by

$$
n c(q)=\frac{36}{\left(2 R_{H S} q\right)^{3}} \frac{\left(1+2 \phi_{\text {disp }}\right)^{2}}{\left(1-\phi_{\text {disp }}\right)^{4}} \phi_{\text {disp }}^{2}\left(x_{1}+x_{2}+x_{2}\right)
$$

with

$$
x_{1}=-2\left(\frac{\sin \left(2 R_{H S} q\right)-2 R_{H S} q \cos \left(2 R_{H S} q\right)}{3 \phi_{\text {disp }}}-\frac{4}{\left(2 R_{H S} q\right)^{3}}\right)
$$




$$
\begin{aligned}
x_{2}=\frac{1}{3}\left(\left(1-\frac{6}{\left(2 R_{H S} q\right)^{2}}\right) 4 \sin \left(2 R_{H S} q\right)\right. \\
\left.\quad\left(1-\frac{12}{\left(2 R_{H S} q\right)^{2}}+\frac{24}{\left(2 R_{H S} q\right)^{4}}\right) 2 R_{H S} q \cos \left(2 R_{H S} q\right)\right) \\
x_{3}=2\left(\left(\frac{2}{\left(2 R_{H S} q\right)^{2}}-1\right) \frac{2 R_{H S} q \cos \left(2 R_{H S} q\right)}{2}+\sin \left(2 R_{H S} q\right)-\frac{1}{2 R_{H S} q}\right)
\end{aligned}
$$

with $R_{\mathrm{HS}}$ being the hard sphere radius of the particles. To account for polydispersity of the particles the averaged structure factor

$$
\overline{S_{P Y}(q)}=\int_{0}^{\infty} d R_{H S} S_{P Y}(q) W\left(R_{H S}, R_{H S, 0}, \sigma_{H S}\right)
$$

was used, which was calculated numerically using Mathematica ${ }^{\circledR} 10$.

\section{Unstable larger water (emulsion) droplets}

As the $q$-range studied does not allow for much details regarding the nature of the emulsion droplets a simple polydisperse droplet model in bulk contrast according to ${ }^{3}$ was used. Here the scattering intensity is given by

$$
I_{\text {drop }}(q)=T(q)\left(t_{1}(q)-t_{2}(q)+t_{3}(q)\right)
$$

with

$$
T(q)=\frac{36 \pi}{3} \phi_{\text {drop }} R_{\text {drop }}^{3} \frac{\left(\Delta \rho_{\text {drop }}\right)^{2}}{\left(q R_{\text {drop }}\right)^{2}} \exp \left(-q^{2} t^{2}\right)
$$




$$
\begin{gathered}
t_{1}(q)=0.5\left(1-\cos \left(2 q R_{\text {drop }}\right)\right) \exp \left(-2 q^{2} \sigma_{\text {drop }}^{2}\right) \\
t_{2}(q)=q\left(R_{\text {drop }} \sin \left(2 q R_{\text {drop }}\right)+2 q \sigma_{\text {drop }}^{2} \cos \left(2 q R_{\text {drop }}\right)\right) \exp \left(-2 q^{2} \sigma_{\text {drop }}^{2}\right) \\
t_{3}(q)=0.5\left(t_{4}(q)+R_{\text {drop }}^{2}+\sigma_{\text {drop }}^{2}+t_{5}(q)+t_{6}(q)\right)
\end{gathered}
$$

which in turn includes

$$
\begin{gathered}
t_{4}(q)=-4 q R_{\text {drop }} \sigma_{\text {drop }}^{2} \sin \left(2 q R_{\text {drop }}\right) \exp \left(-2 q^{2} \sigma_{\text {drop }}^{2}\right) \\
t_{5}(q)=R_{\text {drop }}^{2} \cos \left(2 q R_{\text {drop }}\right) \exp \left(-2 q^{2} \sigma_{\text {drop }}^{2}\right) \\
t_{6}(q)=\sigma_{\text {drop }}^{2} \cos \left(2 q R_{\text {drop }}\right)\left(1-4 q^{2} \sigma_{\text {drop }}^{2}\right) \exp \left(-2 q^{2} \sigma_{\text {drop }}^{2}\right)
\end{gathered}
$$

with $R_{\text {drop }}$ and $\sigma_{\text {drop }}$ being the radius and polydispersity of the emulsion droplet.

\section{Additional figure}

The following figure gives a detailed view of the combination of the scattering contribution stemming from the elongated microemulsion droplets (black dashed line) and the larger emulsion droplets (red dashed line).

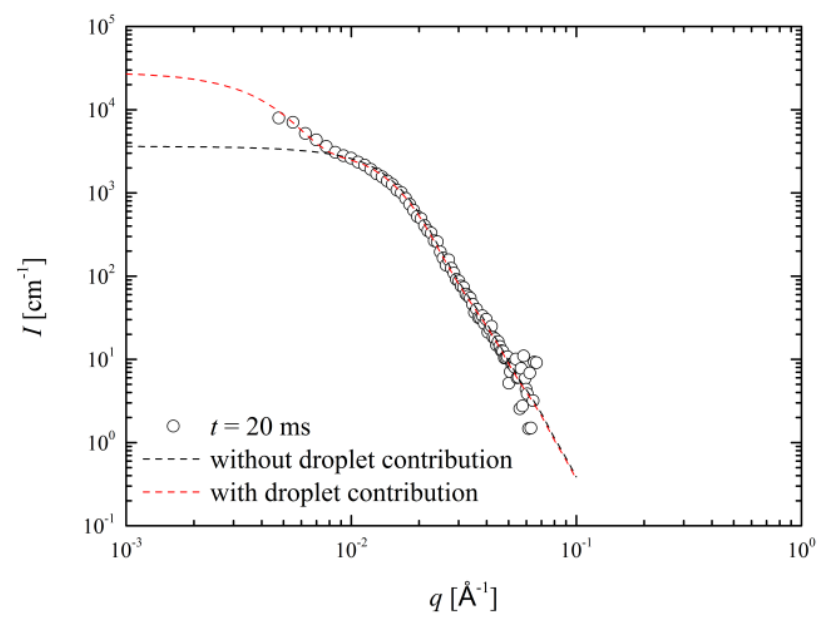


Fig. 1 Comparison of the scattering contributions stemming from emulsion droplets and microemulsion droplets at $t=20 \mathrm{~ms}$ for the data given in the lower part of Fig. 3 in the original document.

\section{References}

1. Foster, T., Universal Analytical Scattering Form Factor for Shell-, Core-Shell, or Homogeneous Particles with Continuously Variable Density Profile Shape. J. Phys. Chem. B 2011, 115 (34), 10207-10217.

2. Foster, T.; Sottmann, T.; Schweins, R.; Strey, R., Small-angle neutron scattering from giant water-in-oil microemulsion droplets. I. Ternary system. J. Chem. Phys. 2008, 128 (5).

3. Gradzielski, M.; Langevin, D.; Magid, L.; Strey, R., Small-angle neutron scattering from diffuse interfaces: 2 . polydisperse shells in water- $n$-alkane- $\mathrm{C}_{10} \mathrm{E}_{4}$ microemulsions. Journal of Physical Chemistry 1995, 99 (35), 13232-13238.

4. Percus, J. K.; Yevick, G. J., Analysis of classical statistical mechanics by means of collective coordinates. Physical Review 1958, 110 (1), 1-13.

5. Foster, T.; Sottmann, T.; Schweins, R.; Strey, R., Small-angle-neutron-scattering from giant water-in-oil microemulsion droplets. II. Polymer-decorated droplets in a quaternary system. J. Chem. Phys. 2008, 128 (6). 